\title{
MAL'TSEV CONDITIONS, LACK OF ABSORPTION, AND SOLVABILITY
}

\author{
LIBOR BARTO, MARCIN KOZIK, DAVID STANOVSKÝ
}

\begin{abstract}
We provide a new characterization of several Mal'tsev conditions for locally finite varieties using hereditary term properties. We show a particular example how lack of absorption causes collapse in the Mal'tsev hierarchy, and point out a connection between solvability and lack of absorption. As a consequence, we provide a new and conceptually simple proof of a result of Hobby and McKenzie, saying that locally finite varieties with a Taylor term possess a term which is Mal'tsev on blocks of every solvable congruence in every finite algebra in the variety.
\end{abstract}

\section{INTRODUCTION}

1.1. Outline. The main purpose of the paper is to relate three important concepts that emerged in universal algebra over the years: the hierarchy of Mal'tsev conditions that has been a central topic in universal algebra since its very beginning [8], the general notion of solvability developed as a part of the commutator theory some 30 years ago [7], and the absorption theory that emerged recently in the context of the algebraic approach to Constraint Satisfaction Problem (CSP) [1].

The paper has three parts. First, we establish alternative characterizations of four important Mal'tsev conditions for idempotent locally finite varieties, namely, the Taylor property, few subpowers, congruence permutability and congruence meet-semidistributivity, using conditions asking for hereditary (non-)existence of certain objects, namely

- non-existence of a cube term blocker,

- existence of a confluent term operation,

- existence of a transitive term operation,

- existence of a weakly pointing term operation, and

- existence of a pointing term operation.

All five conditions are related to the concept of absorption, and they already found, with the exception of confluent operations, some applications. In the second part, we apply the characterizations to show that, in idempotent locally finite varieties, lack of absorption, namely,

- non-existence of absorbing subalgebras, or

- non-existence of bin-absorbing subalgebras,

2010 Mathematics Subject Classification. 08A05, 08B05.

Key words and phrases. Mal'tsev conditions, Taylor term, cube term, Mal'tsev term, Taylor variety, variety with few subpowers, congruence permutable variety, congruence meet-semidistributive variety, absorbing subalgebras, bin-absorbing subalgebras, abelian algebras, solvable algebras, affine algebras.

Research partially supported by the GAČR grant 13-01832S, by National Science Centre based on DEC2011/01/B/ST6/01006, and the Czech-Polish cooperation grant 7AMB13PL013. 
causes a collapse of certain Mal'tsev conditions. We conclude with a proof that solvability (in various forms) causes lack of absorption (on various levels). The three parts combined, we obtain new proofs of some old results of Hobby and McKenzie [9].

We would like to point out that the paper is mostly self-contained, referring only to several simple results on absorption from [1] and to a characterization of congruence meet semidistributive varieties from [9]. In particular, in our proof of the Hobby-McKenzie Theorem 4.7, we avoid references to the tame congruence theory at all.

Now we give necessary definitions and highlight (special cases of) the main results.

\subsection{Hereditary properties and Mal'tsev conditions.}

1.2.1. Hereditary properties propagate. We prove that all the conditions displayed in the outline have a common feature: If a finite idempotent algebra $\mathbf{A}$ and all of its subalgebras satisfy this property then so does every algebra in the pseudovariety generated by $\mathbf{A}$. (Recall that a pseudovariety is a class of algebras closed under homomorphic images, subalgebras, and finitary products.)

Confluent, transitive and (weakly) pointing term operations in the free algebra can be used to obtain terms satisfying certain identities. In this way we obtain new characterizations of varieties with a Taylor term, a cube term (i.e., varieties with few subpowers), and meetsemidistributive varieties (resp.).

1.2.2. Taylor term. An $n$-ary idempotent term $t$ is a Taylor term if, for every coordinate $i \leq n, t$ satisfies an identity of the form

$$
t\left(x_{1}, \ldots, x_{n}\right) \approx t\left(y_{1}, \ldots, y_{n}\right),
$$

where $x_{1}, \ldots, x_{n}, y_{1}, \ldots, y_{n} \in\{x, y\}$ and $x_{i}=x, y_{i}=y$. The existence of a Taylor term is the weakest non-trivial idempotent Mal'tsev condition [20]. Taylor terms recently receive a great deal of attention because of the fact that they are conjectured to distinguish between tractable and NP-complete CSPs [6].

An $n$-ary operation $f$ on a set $A$ is called confluent in $i$-th coordinate, if for every $u, v \in A$ there exist $a_{1}, \ldots, a_{n}, b_{1}, \ldots, b_{n} \in A$ such that $a_{i}=u, b_{i}=v$ and $f\left(a_{1}, \ldots, a_{n}\right)=$ $f\left(b_{1}, \ldots, b_{n}\right)$. Operations which are confluent in every coordinate are called confluent. Our first result connects Taylor terms and confluent operations.

Theorem 1.1. The following are equivalent for a finite idempotent algebra $\mathbf{A}$.

(1) A has a Taylor term;

(2) Every subalgebra of $\mathbf{A}$ has a confluent term operation.

1.2.3. Cube term. An $n$-ary idempotent term $t$ is a cube term if, for every coordinate $i \leq n$, $t$ satisfies an identity of the form

$$
t\left(x_{1}, \ldots, x_{n}\right) \approx y
$$

where $x_{1}, \ldots, x_{n} \in\{x, y\}$ and $x_{i}=x$. Cube terms were independently discovered in [12] and $[4,10]$, in the latter two papers in connection with the property that algebras in the variety have few subpowers and with algorithms for the CSP generalizing Gaussian elimination. A classical example of a cube term is a Mal'tsev term, i.e. a ternary term $m$ satisfying the identities

$$
m(y, x, x) \approx \underset{2}{y} \approx m(x, x, y) .
$$


An analogue of confluent operations are transitive operations. An $n$-ary operation $f$ on a set $A$ is called transitive in $i$-th coordinate, if for every $u, v \in A$ there exist $a_{1}, \ldots, a_{n} \in A$ such that $a_{i}=u$ and $f\left(a_{1}, \ldots, a_{n}\right)=v$. Operations which are transitive in every coordinate are called transitive. Such operations were used in the proof of the Absorption Theorem, one of the key facts in absorption theory [1].

A proper subalgebra $\mathbf{B}$ of an idempotent algebra $\mathbf{A}$ is called a cube term blocker if for every term operation $t$ of $\mathbf{A}$ there exists a coordinate $i$ such that

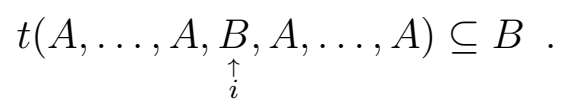

Cube term blockers were introduced in [14] in connection to finitely related algebras and the Valeriote conjecture for congruence modular varieties. ${ }^{1}$

The second major result connects these three concepts.

Theorem 1.2. The following are equivalent for a finite idempotent algebra $\mathbf{A}$.

(1) A has a cube term.

(2) Every subalgebra of $\mathbf{A}$ has a transitive term operation.

(3) No subalgebra of $\mathbf{A}$ has a cube term blocker.

The equivalence $(1) \Leftrightarrow(3)$ was proved in [14], our paper provides an alternative proof. The reader may wonder whether there is a corresponding notion to cube term blockers in the context of Taylor terms. The answer is affirmative, but the characterization we would get is weaker than the one obtained in [5, Proposition 4.14], so we do not formulate it here.

1.2.4. Meet-semidistributivity. A variety is called meet-semidistributive if congruences of all of its members satisfy

$$
\alpha \wedge \beta=\alpha \wedge \gamma \Rightarrow \alpha \wedge(\beta \vee \gamma)=\alpha \wedge \beta
$$

Meet-semidistributive varieties also play a significant role in the CSP where they characterize problems solvable by local propagation methods [2]. There is a number of Mal'tsev conditions characterizing meet-semidistributivity, see e.g. [9, 13, 21].

(Weakly) pointing operations are in a sense dual to transitive operations. An $n$-ary idempotent operation $f$ on $A$ weakly points to $a$ if, for each $j \leq n$, there exists $a_{1}^{j}, \ldots, a_{n}^{j} \in A$ such that

$$
f\left(a_{1}^{j}, \ldots, a_{j-1}^{j}, b, a_{j+1}^{j}, \ldots, a_{n}^{j}\right)=a \text { for all } b \in A .
$$

An $n$-ary idempotent operation $f$ on $A$ points to $a$ if there there exist $a_{1}, \ldots, a_{n} \in A$ such that, for every $j \leq n$,

$$
f\left(a_{1}, \ldots, a_{j-1}, b, a_{j+1}, \ldots, a_{n}\right)=a \text { for all } b \in A .
$$

(Weakly) pointing operations were one of the main algebraic tools in [2]. We prove that pointing operations characterize meet-semidistributivity and use them to give a new Mal'tsev condition:

Theorem 1.3. Let $\mathbf{A}$ be a finite idempotent algebra. The following are equivalent.

(1) A generates a congruence meet-semidistributive variety.

(2) Every subalgebra of $\mathbf{A}$ has a pointing term operation.

\footnotetext{
${ }^{1}$ In [14] a cube term blocker was defined as a pair of subuniverses $(B, C)$. A pair $(B, C)$ is a cube term blocker in their sense iff $B$ is a cube term blocker of $\mathbf{C}$ in our sense.
} 
(3) Every subalgebra of $\mathbf{A}$ has a weakly pointing term operation.

$(4) \mathcal{V}$ has an $n$-ary term $t$ which, for every $i \leq n$, satisfies an identity

$$
t\left(x_{i 1}, x_{i 2}, \ldots, x_{i n}\right) \approx t\left(y_{i 1}, y_{i 2}, \ldots, y_{i n}\right),
$$

where $\left(x_{i j}\right)$ and $\left(y_{i j}\right)$ are $\{x, y\}$-matrices such that $x_{i j}=y_{i j}$ for every $i \neq j$, and $x_{i i}=x, y_{i i}=y$ for every $i$.

In Theorem 2.12, we also show that condition (4) is equivalent to its particular instance, using the matrices

$$
\left(\begin{array}{cccc|cccc}
x & x & \ldots & x & y & y & \ldots & y \\
x & x & \ldots & x & y & y & \ldots & y \\
& & \vdots & & & & \vdots & \\
x & x & \ldots & x & y & y & \ldots & y \\
\hline x & x & \ldots & x & x & y & \ldots & y \\
x & x & \ldots & x & y & x & \ldots & y \\
& & \vdots & & & \vdots & \\
x & x & \ldots & x & y & y & \ldots & x
\end{array}\right), \quad\left(\begin{array}{cccc|cccc}
y & x & \ldots & x & y & y & \ldots & y \\
x & y & \ldots & x & y & y & \ldots & y \\
& & \vdots & & & & \vdots & \\
x & x & \ldots & y & y & y & \ldots & y \\
\hline x & x & \ldots & x & y & y & \ldots & y \\
x & x & \ldots & x & y & y & \ldots & y \\
& & \vdots & & & & \vdots & \\
x & x & \ldots & x & y & y & \ldots & y
\end{array}\right) .
$$

1.3. Lack of absorption and collapses of Mal'tsev conditions. Let B be a subalgebra of $\mathbf{A}$. We say $\mathbf{B}$ is an absorbing subalgebra of $\mathbf{A}$, if there is an idempotent term operation $t$ of $\mathbf{A}$ such that

$$
t(B, \ldots, B, A, B, \ldots, B) \subseteq B
$$

in every coordinate. Such an operation $t$ is called an absorbing term operation for $\mathbf{B}$. An absorbing subalgebra $\mathbf{B}$ of $\mathbf{A}$ is called bin-absorbing if the absorbing term operation for $\mathbf{B}$ can be chosen binary. An algebra $\mathbf{A}$ is called hereditarily absorption-free (shortly, $H A F$ ), if no subalgebra $\mathbf{B} \leq \mathbf{A}$ has a proper absorbing subalgebra, it means, whenever $\mathbf{C}$ is absorbing in $\mathbf{B} \leq \mathbf{A}$, then $C=B$. The notion of hereditarily bin-absorption-freeness (HBAF for short) is defined analogically to HAF-ness.

A very useful property of absorbing subalgebras is that some important properties of subpowers are inherited to them, so it is advantageous when an algebra possesses a nontrivial absorption. Our next result shows that lack of (bin-)absorption has strong consequences, at least for algebras with a Taylor term.

Theorem 1.4. Let $\mathbf{A}$ be a finite idempotent algebra with a Taylor term.

(1) If $\mathbf{A}$ is HAF then it has a Mal'tsev term.

(2) If $\mathbf{A}$ is HBAF then it has a cube term.

Replacing Taylor by a stronger condition, we obtain further collapses as a corollary. For instance, let $\mathbf{A}$ be a finite idempotent algebra. Assume that $\mathbf{A}$ is HAF and generates a congruence meet-semidistributive variety, or assume that $\mathbf{A}$ is HBAF and generates a congruence distributive variety. Then $\mathbf{A}$ has a near unanimity term.

1.4. Solvability and absorption. The last section applies our results to abelian and solvable algebras. We briefly recall basic definitions, see [16] for detailed exposition.

Let $\mathbf{A}$ be an algebra and $\alpha \leq \beta$ its congruences. We say $\beta$ is abelian over $\alpha$ if, for every term operation $t$ and all $(x, y),\left(u_{1}, v_{1}\right), \ldots,\left(u_{n}, v_{n}\right) \in \beta$, the following implication holds:

$$
\left(t\left(x, u_{1}, \ldots, u_{n}\right), t\left(x, v_{1}, \ldots, v_{n}\right)\right) \in \alpha \quad \underset{4}{\Rightarrow}\left(t\left(y, u_{1}, \ldots, u_{n}\right), t\left(y, v_{1}, \ldots, v_{n}\right)\right) \in \alpha .
$$


We say $\beta$ is bin-abelian over $\alpha$, if the condition holds for every binary term operation $t$.

We say $\beta$ is solvable over $\alpha$ (bin-solvable, resp.) if there is a chain of congruences $\alpha=$ $\alpha_{0} \leq \alpha_{1} \leq \ldots \leq \alpha_{n}=\beta$ such that $\alpha_{i}$ is abelian (bin-abelian, resp.) over $\alpha_{i-1}$, for every $i=1, \ldots, n$. A congruence is called abelian (solvable) if it is abelian (solvable) over $0_{A}$. An algebra is called abelian (solvable) if the congruence $1_{A}$ is abelian (solvable). Analogical notions are defined using bin-abelianess and bin-solvability.

Binary versions of abelianess and solvability are rather nonstandard. Note that binabelianess is a strictly weaker notion: e.g., there are non-abelian algebras with no non-trivial binary term operations, such as the two-element algebra with the ternary majority operation.

The following theorem shows a connection to HAF and HBAF algebras.

\section{Theorem 1.5.}

(1) Finite solvable algebras are $H A F$.

(2) Finite bin-solvable algebras are $H B A F$.

The converse of Theorem 1.5 fails, even for idempotent algebras: there are non-solvable idempotent HAF algebras, e.g., the idempotent reduct of the group $\mathbf{A}_{5}$ [17].

As a consequence we get an alternative proof of (a part) of [9, Theorem 9.6]. The second corollary concerning bin-solvability is, we believe, new.

\section{Corollary 1.6.}

(1) Finite solvable algebras with a Taylor term have a Mal'tsev term.

(2) Finite bin-solvable algebras with a Taylor term have a cube term.

Proof. Let $\mathbf{A}$ be a finite solvable algebra with a Taylor term and consider its idempotent reduct $\mathbf{A}^{I}$. It is solvable, too, it retains the Taylor term, so Theorems 1.4 and 1.5 apply and we obtain a Mal'tsev term for $\mathbf{A}^{I}$, and thus for $\mathbf{A}$, too. Similarly for bin-solvability.

We cannot drop the assumption of finiteness in Theorem 1.4 and Corollary 1.6:

Example 1. Let $\mathbf{A}=(\mathbb{Q}, F)$ where $F$ consists of all binary operations $x *_{a} y=(1-a) x+a y$ with $a \in(0,1) \cap \mathbb{Q}$. This is an abelian idempotent algebra with a binary Taylor term $*_{1 / 2}$. It has no cube term, because all operations $*_{a}$ preserve the natural order of $\mathbb{Q}$. Hence Corollary 1.6 fails for infinite algebras. Now $B \subseteq \mathbb{Q}$ forms a subalgebra of $\mathbf{A}$ if and only if $B$ is an interval, hence $\mathbf{A}$ is HAF. Thus Theorem 1.4 fails for infinite algebras.

On the other hand, the relation of solvability (or abelianess) and absorption-freeness is unclear for infinite algebras. Some thoughts with respect to extending Theorem 1.5 towards infinite algebras are presented in the last section. Consequences for affine representations are also discussed there (comp. [18, 19] for background).

\section{Hereditary properties And MaL'TSEV CONDitions}

We first introduce useful notation. When $f$ is an $m$-ary term and $g_{1}, \ldots, g_{m}$ are $n$-ary terms, by $f\left[g_{1}, \ldots, g_{m}\right]$ we mean the $m n$-ary term

$$
f\left(g_{1}\left(x_{11}, \ldots, x_{1 n}\right), g_{2}\left(x_{21}, \ldots, x_{2 n}\right), \ldots, g_{m}\left(x_{m 1}, \ldots, g_{m n}\right)\right) .
$$

We define $f * g:=f[g, g, \ldots, g]$. 


\subsection{Hereditary properties propagate.}

Proposition 2.1. The following classes of idempotent algebras (of a fixed signature) are pseudovarieties:

(1) $\mathcal{K}=\{\mathbf{A}: \mathbf{A}$ is $H A F\}$

(2) $\mathcal{K}=\{\mathbf{A}: \mathbf{A}$ is $H B A F\}$

(3) $\mathcal{K}=\{\mathbf{A}:$ no subalgebra of $\mathbf{A}$ has a cube term blocker $\}$

(4) $\mathcal{K}=\{\mathbf{A}: \mathbf{A}$ is finite and every subalgebra of $\mathbf{A}$ has a confluent term operation $\}$

(5) $\mathcal{K}=\{\mathbf{A}: \mathbf{A}$ is finite and every subalgebra of $\mathbf{A}$ has a transitive term operation $\}$

(6) $\mathcal{K}=\{\mathbf{A}: \mathbf{A}$ is finite and every subalgebra of $\mathbf{A}$ has a weakly pointing term operation $\}$

Proof. All classes are trivially closed under subalgebras and, in cases (3)-(6) under quotients. In cases (1) and (2) the class $\mathcal{K}$ is also closed under quotients since union of blocks in an absorbing subuniverse of a quotient is an absorbing subuniverse in the original algebra. Therefore we can concentrate on products.

The proofs for (1), (2) and (3) are similar. We only prove (1). Let $\mathbf{A}$ and $\mathbf{B}$ be HAF and let $\mathbf{D}$ be an absorbing subalgebra of $\mathbf{C} \leq \mathbf{A} \times \mathbf{B}$, with absorbing term operation $t$. It is clear that $\operatorname{proj}_{1}(\mathbf{D})$ absorbs $\operatorname{proj}_{1}(\mathbf{C})$ and $\operatorname{proj}_{2}(\mathbf{D})$ absorbs $\operatorname{proj}_{2}(\mathbf{C}$ ) (with absorbing term operation $t$ ) and, since $\mathbf{A}$ and $\mathbf{B}$ are HAF, both projections of $\mathbf{D}$ and $\mathbf{C}$ are equal. Suppose, for a contradiction, that $D \neq C$, hence we can choose $a \in A$ such that $B^{\prime}=\{b \in B$ : $(a, b) \in D\} \varsubsetneqq\{b \in B:(a, b) \in C\}=B^{\prime \prime}$. Since $\mathbf{D}$ and $\mathbf{C}$ are idempotent, both $\mathbf{B}^{\prime}$ and $\mathbf{B}^{\prime \prime}$ are subalgebras of $\mathbf{B}$. Clearly $\emptyset \neq B \mp B^{\prime}$ and $\mathbf{B}$ is absorbing in $\mathbf{B}^{\prime}$ (with absorbing term operation $t$ ). This contradicts the assumption that $\mathbf{B}$ was HAF.

The proofs for (4), (5) and (6) are similar. We only prove (5). Let $\mathbf{A}$ and $\mathbf{B}$ be algebras in $\mathcal{K}$, and define $t_{A}\left(t_{B}\right.$, respectively) as $t_{1} * t_{2} * \ldots$, where $t_{1}, t_{2}, \ldots$ is a list of transitive term operations for all subalgebras of $\mathbf{A}$ ( $\mathbf{B}$, respectively). The operation $t_{A}\left(t_{B}\right)$ is a transitive term operation in every subalgebra of $\mathbf{A}(\mathbf{B})$. We claim that $t=t_{B} * t_{A}$ is transitive in every subalgebra $\mathbf{C} \leq \mathbf{A} \times \mathbf{B}$. Take any coordinate $i$ of $t$ and any $u=(a, b), v=\left(a^{\prime}, b^{\prime}\right) \in C$. We need to find $\left(a_{1}, b_{1}\right), \ldots \in C$ such that $a_{i}=a, b_{i}=b$, and $t\left(\left(a_{1}, b_{1}\right), \ldots\right)=\left(a^{\prime}, b^{\prime}\right)$. The pairs within the term operation $t_{A}$ containing the $i$-th coordinate are chosen so that the result of $t_{A}$ is of the form $\left(a^{\prime}, b^{\prime \prime}\right)$ for some $b^{\prime \prime} \in B$ (we can do that since $t_{A}$ is transitive on $\operatorname{proj}_{1}(\mathbf{C})$ ). In every other copy of $t_{A}$, the variables are set to the same element of the form $\left(a^{\prime}, b_{j}\right) \in C$. Since $t_{B}$ is transitive on $\left\{b \in B:\left(a^{\prime}, b\right) \in C\right\}$, we can do it so that $t_{B}$ applied to the results of $t_{A}$ 's is equal to $\left(a^{\prime}, b^{\prime}\right)$.

The corresponding statement for pointing operations follows from Theorem 2.12.

2.2. Taylor term. We prove a generalization of Theorem 1.1 characterizing Taylor terms by means of confluent operations. The core of the argument is the following lemma.

Lemma 2.2. Let $\mathcal{V}$ be an idempotent variety. If the free algebra on two generators in $\mathcal{V}$ has a confluent term operation than $\mathcal{V}$ has a Taylor term.

Proof. Let $t$ be a confluent term operation of $\mathbf{F}$. Let $f_{i}^{j}$, s and $g_{i}^{j}$, s be elements of $\mathbf{F}$ such that for every $j$

$$
t\left(f_{1}^{j}, \ldots, f_{n}^{j}\right)=t\left(g_{1}^{j}, \ldots, g_{n}^{j}\right) \text { and } f_{j}^{j}=x, g_{j}^{j}=y .
$$

Let $h_{i}=f_{i}^{1} * \cdots * f_{i}^{n} * g_{i}^{1} * \cdots * g_{i}^{n}$. Note that each $f_{i}^{j}$ and $g_{i}^{j}$ can be obtained from $h_{i}$ by setting a set of variables of $h_{i}$ to $x$ and the rest of the variables to $y$ (we are using idempotency here). Now we show that $s=t\left[h_{1}, \ldots, h_{n}\right]$ is a Taylor term. 
To do so, we fix a coordinate $i$ and attempt to fill in remaining coordinates so that

$$
s(\ldots, \underset{\substack{\uparrow \\ i}}{x}, \ldots) \approx s(\ldots, \underset{\uparrow}{y}, \ldots)
$$

holds. Suppose $i$ appears within the scope of $h_{j}$. We choose all the variables within $h_{j}$ to evaluate to $x$ on the left ( $h_{j}$ evaluates to $x$ on the left) and to $y$ on the right. We identify the variables in all other $h_{k}$ 's to obtain $f_{k}^{j}$ 's on the left and $g_{k}^{j}$ 's on the right. By construction, the evaluations of $s$ on the left and on the right are equal.

Theorem 2.3. Let $\mathcal{A}$ be a set of idempotent algebras, and assume that the variety $\mathcal{V}$ generated by $\mathcal{A}$ is locally finite. The following are equivalent.

(1) $\mathcal{V}$ has a Taylor term;

(2) every finite subalgebra of an algebra in $\mathcal{A}$ has a confluent term operation.

Proof. The implication (1) $\Rightarrow(2)$ is trivial since a Taylor term operation is clearly confluent.

To prove $(2) \Rightarrow(1)$, consider the free algebra $\mathbf{F}$ on two generators in $\mathcal{V}$. Since it is finite, it lies in the pseudovariety generated by finite subalgebras of algebras from $\mathcal{A}$. Then $\mathbf{F}$ is confluent by Proposition 2.1.(4) and $\mathcal{V}$ has a Taylor term by Lemma 2.2.

An easy consequence of Theorem 2.3 is the following.

Corollary 2.4. Let $\mathbf{A}$ be a finite idempotent algebra. If every 2-generated subalgebra of $\mathbf{A}$ has a Taylor term then so does $\mathbf{A}$.

Proof. Since every 2-generated subalgebra of $\mathbf{A}$ has a Taylor term it also has a confluent term operation. Then, for every $u, v \in A$, considering the subalgera generated by $u, v$, we obtain a term operation $t_{u, v}$ of $\mathbf{A}$ satisfying the requirement for a confluent operation for the pair $(u, v)$. Take a list $t_{1}, \ldots, t_{k}$ containing such "partially confluent" operations for every pair $(u, v) \in A^{2}$. Now $t=t_{1} * \cdots * t_{k}$ is a confluent term operation in every subalgebra of A, so $\mathbf{A}$ has a Taylor term.

2.3. Cube term. Next we prove a generalization of Theorem 1.2 relating cube terms, cube term blockers and transitive operations. We start with an analogue of Lemma 2.2 for transitive operations. The proof is similar.

Lemma 2.5. Let $\mathcal{V}$ be an idempotent variety. If the free algebra on two generators has a transitive term operation then $\mathcal{V}$ has a cube term.

In [1], the main auxiliary result for the Absorption Theorem was Proposition 2.7 which proves the existence of transitive operations. Minor changes of the proof gives us the implication "no blocker $\Rightarrow$ transitive".

Lemma 2.6. Let $\mathbf{A}$ be a finite idempotent algebra. If $\mathbf{A}$ has no cube term blocker then $\mathbf{A}$ has a transitive term operation.

Proof. For a term operation $t$ of $\mathbf{A}$ of arity $k$, an element $b \in A$, and a coordinate $i \leq \operatorname{arity}(t)$ we set

$$
W(t, b, i)=\left\{t\left(a_{0}, \ldots, a_{k-1}\right): a_{i}=b \text { and } \forall j a_{j} \in A\right\} .
$$

Our aim is to find a term operation $v$ such that $W(v, b, i)=A$ for any $b \in A$ and any coordinate $i$. We will achieve this goal by gradually enlarging the sets $W(t, b, i)$. 
Let $n<|A|$ and assume we have already found term operations $v^{(n)}$ such that each $W\left(v^{(n)}, b, i\right)$ contains a subuniverse of $\mathbf{A}$ with at least $n$ elements. From idempotency it follows that all the one-element subsets of $A$ are subuniverses of $\mathbf{A}$, thus any term operation can be taken as $v^{(1)}$.

For an induction step we first find a term operation $w^{(n+1)}$ such that each $W\left(w^{(n+1)}, b, i\right)$ has at least $(n+1)$-elements. Let $B_{1}, B_{2}, \ldots, B_{l}$ be the list of all at most $n$-element subuniverses of $\mathbf{A}$. For every $i \leq l$ there exists a term operation $t_{i}$ witnessing the fact that $\left(B_{i}, A\right)$ is not a cube term blocker, that is, $B_{i} \subsetneq t_{i}\left(A, \ldots, A, B_{i}, A, \ldots, A\right)$ for every coordinate. Then the term operation $t=t_{1} * t_{2} * \cdots * t_{l}$ is a common witness, i.e. for all $i \leq l$ we have $B_{i} \subsetneq t\left(A, \ldots, A, B_{i}, A, \ldots, A\right)$ for any position of $B_{i}$. Now we can put $w^{(n+1)}=t * v^{(n)}$.

The proof can be finished in the same way as in [1]. The term operation $v^{(n)}$ is constructed in Claim 2.9 within Proposition 2.7 and we take $v=v^{|A|}$.

Theorem 2.7. Let $\mathcal{A}$ be a set of idempotent algebras, and assume that the variety $\mathcal{V}$ generated by $\mathcal{A}$ is locally finite. The following are equivalent.

(1) $\mathcal{V}$ has a cube term.

(2) every finite subalgebra of an algebra in $\mathcal{A}$ has a transitive term operation.

(3) no proper subalgebra of an algebra in $\mathcal{A}$ has a cube term blocker.

Proof. The implications $(1) \Rightarrow(2),(3)$ are trivial.

To prove $(3) \Rightarrow(1)$ we consider (as in the proof of Theorem 2.3) the free algebra $\mathbf{F}$ on two generators in $\mathcal{V}$. Since it is finite, it lies in the pseudovariety generated by finite subalgebras of algebras from $\mathcal{A}$. By Proposition 2.1.(3), F has no cube term blocker and then, by Lemma 2.6, $\mathbf{F}$ has a transitive term operation. Now $\mathcal{V}$ has a cube term by Lemma 2.5.

The proof $(2) \Rightarrow(1)$ is similar, using Proposition 2.1.(5) and Lemma 2.5.

We also have a similar consequence.

Corollary 2.8. Let $\mathbf{A}$ be a finite idempotent algebra. If every 2-generated subalgebra of $\mathbf{A}$ has a cube term then so does $\mathbf{A}$.

2.4. Meet-semidistributivity. The last Mal'tsev condition considered in this section is conguence meet-semidistributivity. The most difficult step for the characterization is to prove that finite idempotent algebras in congruence meet-semidistributive varieties have pointing term operations. A partial result in this direction was proved in [2].

Lemma 2.9. Let $\mathbf{A}$ be a simple idempotent algebra with no proper absorbing subuniverse generating a meet-semidistributive variety. For every $a \in A$ there exists a term operation of A which points to a.

In the general case we proceed by induction on the size of $\mathbf{A}$. The following lemma shows that we can assume that $\mathbf{A}$ is absorption-free.

Lemma 2.10. Let $\mathbf{A}$ be an idempotent algebra and $\mathbf{B}$ an absorbing subalgebra of $\mathbf{A}$. If $\mathbf{B}$ has a pointing term operation then so does $\mathbf{A}$.

Proof. Let $w$ be an $l$-ary term operation providing the absorption and let $t$ be a $k$-ary term operation of $\mathbf{B}$ which points to $b$ using the tuple $\left(b_{1}, \ldots, b_{k}\right)$. Then the term operation $t * w$ of the algebra A points to $b$ using the tuple

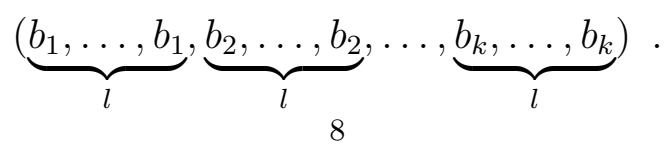


Lemma 2.11. Let $\mathbf{A}$ be a finite idempotent algebra in a congruence meet-semidistributive variety. Then $\mathbf{A}$ has a term operation pointing to one of its elements.

Proof. The proof is by induction on $|\mathbf{A}|$, thus we assume that the lemma holds for all the algebras with fewer than $|\mathbf{A}|$ elements. By Lemma 2.10, $\mathbf{A}$ is absorption-free.

We take a maximal non-trivial congruence $\alpha$. Lemma 2.9 provides existence of term operations of $\mathbf{A} / \alpha$ pointing to $a / \alpha$ for any $a$.

Claim: there exists a $k$-ary term operation of $\mathbf{A} / \alpha$ which points using the tuple $a_{1} / \alpha, \ldots, a_{k} / \alpha$ such that for every $i$

$$
\left\{t\left(b_{1}, \ldots, b_{k}\right): \forall j \neq i\left(b_{j}, a_{j}\right) \in \alpha\right\}=\left\{t\left(b_{1}, \ldots, b_{k}\right): \forall j\left(b_{j}, a_{j}\right) \in \alpha\right\} .
$$

To prove the claim we introduce some notation. For a $n$-ary term operation $r$ in $\mathbf{A} / \alpha$ which points using the tuple $\left(a_{1} / \alpha, \ldots, a_{n} / \alpha\right)$ we define

$$
\chi_{r,\left(a_{1}, \ldots, a_{n}\right)}=\max _{i}\left|\left\{r\left(b_{1}, \ldots, b_{n}\right): \forall j \neq i\left(b_{j}, a_{j}\right) \in \alpha\right\}\right| .
$$

Now let $t$ be a $k$-ary term operation of $\mathbf{A} / \alpha$ which points using the tuple $\left(a_{1} / \alpha, \ldots, a_{k} / \alpha\right)$ chosen in such a way that $\chi_{t,\left(a_{1}, \ldots, a_{k}\right)}$ is minimal. We will show that

$$
\chi_{t,\left(a_{1}, \ldots, a_{k}\right)}=\left|\left\{r\left(b_{1}, \ldots, b_{k}\right): \forall j\left(b_{j}, a_{j}\right) \in \alpha\right\}\right|,
$$

which will prove that $t$ satisfies the conditions of the claim.

Let $r_{1}, \ldots, r_{k}$ be terms operation of $\mathbf{A} / \alpha$ pointing to $a_{1} / \alpha, \ldots, a_{k} / \alpha$, respectively. Take $t^{\prime}=t\left[r_{1}, \ldots, r_{k}\right]$. Let $\left(a_{1}^{\prime}, \ldots, a_{m}^{\prime}\right)$ be the tuple obtained from concatenating tuples which witness that $r_{i}$ points to $a_{i} / \alpha$. The term operation $t^{\prime}\left(x_{1}, \ldots, x_{m}\right)$ points in $\mathbf{A} / \alpha$ (using this tuple) to the same element that $t$ was pointing to.

Moreover

$$
\begin{aligned}
\left|\left\{t\left(b_{1}, \ldots, b_{k}\right): \forall j\left(b_{j}, a_{j}\right) \in \alpha\right\}\right| \leq \chi_{t,\left(a_{1}, \ldots, a_{k}\right)} \leq \\
\quad \leq \chi_{t^{\prime},\left(a_{1}^{\prime}, \ldots, a_{m}^{\prime}\right)} \leq\left|\left\{t\left(b_{1}, \ldots, b_{k}\right): \forall j\left(b_{j}, a_{j}\right) \in \alpha\right\}\right|
\end{aligned}
$$

where the first inequality follows from the definition of $\chi$, the second one from minimality of $\chi_{t, a_{1}, \ldots, a_{k}}$ and the third one from construction of $t^{\prime}$. This proves the claim.

From this point on we fix term operation $t$ and the tuple $\left(a_{1}, \ldots, a_{k}\right)$ provided by the claim. Let

$$
B^{\prime}:=t\left(a_{1} / \alpha, \ldots, a_{k} / \alpha\right) \subseteq A \text { and } \mathbf{B}=\operatorname{Sg}_{\mathbf{A}}\left(B^{\prime}\right) .
$$

Since $t$ is pointing in $\mathbf{A} / \alpha$ using $\left(a_{1}, \ldots, a_{k}\right)$, the algebra $\mathbf{B}$ lies fully in one congruence block of $\alpha$. Therefore, by inductive assumption, we get an $l$-ary term operation $r$ of $\mathbf{B}$ which is pointing using a tuple $\left(b_{1}, \ldots, b_{l}\right)$.

Let $s_{i}$ be a term operation generating $b_{i}$ from elements of $B^{\prime}$. We put $s_{i}^{\prime}=s_{i} * t$ and claim that the term operation $r^{\prime}=r\left[s_{1}^{\prime}, \ldots, s_{l}^{\prime}\right]$ of $\mathbf{A}$ is pointing.

We begin to construct the tuple which shows that $r^{\prime}$ is pointing. Focusing within $s_{1}$ we let $c_{1}, \ldots, c_{m} \in B^{\prime}$ be such that $s_{1}\left(c_{1}, \ldots, c_{m}\right)=b_{1}$. By definition of $B^{\prime}$ we get $d_{i}^{j}, i \leq m, j \leq k$

$$
\forall i t\left(d_{i}^{1}, \ldots, d_{i}^{k}\right)=c_{i} \text { and } \forall i, j\left(d_{i}^{j}, a_{j}\right) \in \alpha .
$$

The term operation $s^{\prime}=s_{i} * t$ applied to the tuple

$$
\left(d_{1}^{1}, \ldots, d_{1}^{k}, d_{2}^{1}, \ldots, d_{2}^{k}, \ldots, d_{m}^{1}, \ldots, d_{m}^{k}\right)
$$


produces $b_{1}$. Moreover, substituting any coordinate of this tuple with an arbitrary element of A we obtain, from the properties of $t$ and the construction of $s^{\prime}$, a result in $\mathbf{B}$. By repeating this procedure for all the $c_{i}$ 's and concatenating obtained tuples we get a tuple witnessing that $r^{\prime}$ is a pointing term operation.

We are ready to state a generalization of Theorem 1.3.

Theorem 2.12. Let $\mathcal{A}$ be a set of idempotent algebras, and assume that the variety $\mathcal{V}$ generated by $\mathcal{A}$ is locally finite. Then the following are equivalent.

(1) $\mathcal{V}$ is congruence meet-semidistributive.

(2) every finite subalgebra of an algebra in $\mathcal{A}$ has a pointing term operation.

(3) every finite subalgebra of an algebra in $\mathcal{A}$ has a weakly pointing term operation.

(4) $\mathcal{V}$ has an $n$-ary term $t$ which, for every $i \leq n$, satisfies an identity

$$
t\left(x_{i 1}, x_{i 2}, \ldots, x_{i n}\right) \approx t\left(y_{i 1}, y_{i 2}, \ldots, y_{i n}\right),
$$

where $\left(x_{i j}\right)$ and $\left(y_{i j}\right)$ are $\{x, y\}$-matrices such that $x_{i j}=y_{i j}$ for every $i \neq j$, and $x_{i i}=x, y_{i i}=y$ for every $i$.

(5) $\mathcal{V}$ has a 2 -ary idempotent term $t$ such that

$$
\begin{aligned}
& t(\underbrace{y, x, \ldots, x}_{n}, \underbrace{y, \ldots, y}_{n}) \approx t(\underbrace{x, y, x \ldots, x}_{n}, \underbrace{y, \ldots, y}_{n}) \approx \cdots \approx t(\underbrace{x, \ldots, x, y}_{n}, \underbrace{y, \ldots, y}_{n}) \approx \\
& t(\underbrace{x, \ldots, x}_{n}, \underbrace{y, \ldots, y}_{n}) \approx \\
& t(\underbrace{x, \ldots, x}_{n}, \underbrace{x, y, \ldots, y}_{n}) \approx t(\underbrace{x, \ldots, x}_{n}, \underbrace{y, x, y, \ldots, y}_{n}) \approx \cdots \approx t(\underbrace{x, \ldots, x}_{n}, \underbrace{y, \ldots, y, x}_{n}) .
\end{aligned}
$$

Proof. (1) $\Rightarrow$ (2) follows from Lemma 2.11. (2) $\Rightarrow$ (3) is trivial.

$(3) \Rightarrow(4)$. Let $\mathbf{F}$ be the free algebra in $\mathcal{V}$ generated by $x, y$. Since $\mathbf{F}$ is finite, it belongs to the pseudovariety generated by finite subalgebras of algebras from $\mathcal{A}$. Then $\mathbf{F}$ has a weakly pointing term operation $t^{\prime}$ by Proposition 2.1.(6). Let $f_{i}^{j}$ be the elements of $\mathbf{F}$ which witness that $t^{\prime}$ is a weakly pointing term operation, and set $f_{i}=f_{i}^{1} * f_{i}^{2} * \ldots * f_{i}^{\text {arity }\left(t^{\prime}\right)}$. Then the term $t=t^{\prime}\left[f_{1}, \ldots, f_{n}\right]$ satisfies the required identities by a reasoning similar to the proof of Lemma 2.2 .

$(1) \Rightarrow(5)$. Let again $\mathbf{F}$ be the free algebra in $\mathcal{V}$ generated by $x, y$. By Lemma $2.11, \mathbf{F}$ has a term operation $t^{\prime}$ pointing to some element. This fact is witnessed by elements $f_{1}, \ldots, f_{n} \in \mathbf{F}$. Let $t^{\prime \prime}=t^{\prime}\left[f_{1}, \ldots, f_{n}\right]$. After permuting variables (putting all odd coordinates first) the term $t$ obtained from $t^{\prime \prime}$ satisfies the required identities (and some extra ones).

$(4) \Rightarrow(1),(5) \Rightarrow(1)$. By [9, Theorem 9.10], $\mathcal{V}$ is meet-semidistributive if it satisfies an idempotent Mal'tsev condition which is not satisfiable in any vector space over a field. This condition is easily checked in both cases.

An interesting consequence of Theorem 2.12 and Lemma 2.10 is the following.

Corollary 2.13. Let $\mathbf{A}$ be a finite idempotent algebra. If every absorption-free subalgebra of $\mathbf{A}$ generates a meet-semidistributive variety then so does $\mathbf{A}$.

\section{LACK OF ABSORPTION AND COLLAPSES OF MAL'TSEV CONDITIONS}

In this section we prove a generalization of Theorem 1.4. 
3.1. HAF and Taylor $\Rightarrow$ Mal'tsev. For the first part we use a key fact from the absorption theory [1].

Lemma 3.1 (Absorption Theorem [1, Theorem 2.3]). Let A, B be finite idempotent algebras with Taylor terms, let $R$ be a subdirect subuniverse of $\mathbf{A} \times \mathbf{B}$, and let $\pi_{A}$ ( $\pi_{B}$, resp.) be the kernel of the projection $R \rightarrow A\left(R \rightarrow B\right.$, resp.). If $\pi_{A} \vee \pi_{B}=1_{R}$ then either $R=A \times B$, or A has a proper absorbing subuniverse, or $\mathbf{B}$ has a proper absorbing subuniverse.

Theorem 3.2. Let $\mathcal{V}$ be a locally finite variety generated by a set $\mathcal{A}$ of idempotent HAF algebras. If $\mathcal{V}$ has a Taylor term then it has a Mal'tsev term.

Proof. Let $\mathbf{F}$ be the free algebra on two generators $x, y$ in the variety $\mathcal{V}$. Since it is finite, it lies in the pseudovariety generated by $\mathcal{A}$, and thus it is absorption-free by Proposition 2.1.(1). Let $\mathbf{R}$ be the subalgebra of $\mathbf{F}^{2}$ generated by $(x, y),(x, x),(y, x)$. It is subdirect in $\mathbf{F}^{2}$. Since $\mathbf{F}$ is idempotent, $\pi_{A} \vee \pi_{B}=1_{R}$. Consequently, using Lemma 3.1, $\mathbf{R}=\mathbf{F}^{2}$, and there is a term $m$ witnessing that $(y, y) \in R$, i.e., a term $m$ satisfying $m((x, y),(x, x),(y, x))=(y, y)$. This term is a Mal'tsev term for $\mathcal{V}$.

By applying Theorem 3.2 to the variety generated by all HAF algebras in a variety, we get a useful consequence:

Corollary 3.3. Let $\mathcal{V}$ be a locally finite idempotent variety with a Taylor term. Then there is a term that is a Mal'tsev term on all HAF algebras in $\mathcal{V}$.

3.2. HBAF and Taylor $\Rightarrow$ cube. For the binary version of Theorem 1.4 we use an auxiliary result which was implicitly contained in [1, Proposition 2.7].

Lemma 3.4. Let $\mathbf{A}$ be an idempotent bin-absorption-free algebra with a Taylor term. Then A has no cube term blocker.

Proof. Let $t$ be an $n$-ary Taylor term of $\mathbf{A}$ and assume that $B$ is a cube term blocker. Then there exists a coordinate $i$ such that $t\left(a_{1}, \ldots, a_{n}\right) \in B$ whenever $a_{i} \in B$. Since $t$ is a Taylor term, $t$ satisfies an identity of the form

$$
t\left(x_{1}, \ldots, x_{n}\right) \approx t\left(y_{1}, \ldots, y_{n}\right),
$$

where $x_{1}, \ldots, x_{n}, y_{1}, \ldots, y_{n} \in\{x, y\}$ and $x_{i}=x, y_{i}=y$.

We claim that the binary term operation $f(x, y)=t\left(x_{1}, \ldots, x_{n}\right)=t\left(y_{1}, \ldots, y_{n}\right)$ is an absorbing term operation for $B$. Indeed, if $b \in B, a \in A$ then, since $f(x, y)=t\left(x_{1}, \ldots, x_{n}\right)$, we have $f(b, a)=t\left(c_{1}, \ldots, c_{n}\right)$, where $c_{k} \in\{a, b\}$ and $c_{k}=b$ iff $x_{k}=x$ for every $k$. As $a_{i}=b$ it follows that $f(b, a) \in B$. On the other hand, from $f(x, y)=t\left(y_{1}, \ldots, y_{k}\right)$ we get $t(a, b)=t\left(d_{1}, \ldots, d_{n}\right)$, where $d_{k} \in\{a, b\}$ and $d_{k}=a$ iff $y_{k}=x$ for every $k$. Then $d_{i}=b$ and $t(a, b) \in B$.

Theorem 3.5. Let $\mathcal{V}$ be a locally finite variety generated by a set of idempotent HBAF algebras. If $\mathcal{V}$ has a Taylor term then it has a cube term.

Proof. By Proposition 2.1.(2), the free algebra $\mathbf{F}$ on two generators in $\mathcal{V}$ is HBAF. From Lemma 3.4 it follows that $\mathbf{F}$ has no cube term blocker. Now $\mathbf{F}$ has a transitive term operation by Lemma 2.6, and then $\mathcal{V}$ has a cube term by Lemma 2.5 .

We state a consequence analogous to Corollary 3.3.

Corollary 3.6. Let $\mathcal{V}$ be a locally finite idempotent variety with a Taylor term. Then there is a term that is a cube term on all HBAF algebras in $\mathcal{V}$. 
3.3. $\alpha$-absorption. Next we define a finer notion of absorption which will be used for the application in solvable algebras.

Let $\alpha$ be a congruence of $\mathbf{A}$. For $X \subseteq A$ we denote $X^{\alpha}=\{a \in A:(a, x) \in \alpha$ for some $x \in X\}$. Let $\mathbf{B}$ be a subalgebra of $\mathbf{A}$. We say $\mathbf{B}$ is an $\alpha$-absorbing subalgebra of $\mathbf{A}$ if $\mathbf{B}$ absorbs $\mathbf{B}^{\alpha}$. The original notion of absorption refers to $\alpha=1_{A}$. An algebra $\mathbf{A}$ is called hereditarily $\alpha$-absorption free (shortly, $\alpha-H A F$ ), if no subalgebra $\mathbf{B} \leq \mathbf{A}$ has a proper $\left.\alpha\right|_{B}$-absorbing subalgebra, it means, whenever $\mathbf{C}$ is $\alpha$-absorbing in $\mathbf{B} \leq \mathbf{A}$, then $C=C^{\left.\alpha\right|_{B}}$. Similarly for bin-absorption.

Theorem 3.7. Let $\mathbf{A}$ be a finite idempotent algebra with a Taylor term and $\alpha$ a congruence of $\mathbf{A}$.

(1) If $\mathbf{A}$ is $\alpha-H A F$, then $\mathbf{A}$ has a term that is a Mal'tsev term on every block of $\alpha$.

(2) If $\mathbf{A}$ is $\alpha-H B A F$, then $\mathbf{A}$ has a term that is a cube term on every block of $\alpha$.

Proof. In view of Corollaries 3.3 and 3.6, it is sufficient to show that all blocks of $\alpha$ are HAF (HBAF, resp.). Assume $\mathbf{C}$ is a (bin-)absorbing subalgebra of a subalgebra $\mathbf{B}$ of a block of $\alpha$, with an absorbing term operation $t$. Then $\mathbf{B}$ is a subalgebra of $\mathbf{A}$, too, $\left.\alpha\right|_{B}=1_{B}$, and thus $t\left(C, \ldots, C, C^{\left.\alpha\right|_{B}}, C, \ldots, C\right)=t(C, \ldots, C, B, C, \ldots, C) \subseteq B$, in every coordinate. It follows from $\alpha$-absorption-freeness that $C=C^{\left.\alpha\right|_{B}}=B$.

\section{Solvability AND ABSORPtion}

4.1. (Bin-)solvable $\Rightarrow \mathbf{H}(\mathbf{B}) \mathbf{A F}$. We first aim to show that solvable (bin-solvable, resp.) algebras are HAF (HBAF, resp.). We start with the abelian case.

Lemma 4.1. Let $\mathbf{A}$ be a finite idempotent algebra and $\alpha$ an abelian (bin-abelian, resp.) congruence of $\mathbf{A}$. Then $\mathbf{A}$ does not have a proper $\alpha$-absorbing ( $\alpha$-bin-absorbing, resp.) subalgebra.

Proof. Let $\mathbf{B}$ be an $\alpha$-absorbing subalgebra of $\mathbf{A}$, with an absorbing term operation $t$. (In the bin-abelian case, consider $t$ at most binary.) We show that $B=B^{\alpha}$.

First, assume that $B^{2} \subseteq \alpha$. We proceed by induction on the arity of $t$. If $t$ is unary, it is the identity mapping, and thus $B=t\left(B^{\alpha}\right)=B^{\alpha}$. In the induction step, we prove that if $t$ is an $n$-ary absorbing term operation, then there also exists an $(n-1)$-ary absorbing term operation for $\mathbf{B}$. Let $\bar{x}$ denote the $(n-1)$-tuple $x_{1}, \ldots, x_{n-1}$ and put

$$
t_{m}(\bar{x}, y)=\underbrace{t(\bar{x}, t(\bar{x}, t(\ldots, t(\bar{x}}_{m}, y)))) \text {. }
$$

Then $t_{m}$ is also an absorbing term operation for $\mathbf{B}$. Choose $m$ such that

$$
t_{m}\left(\bar{x}, t_{m}(\bar{x}, y)\right)=t_{m}(\bar{x}, y)
$$

(for instance, $m=|A|$ ! will do). We show that the term operation

$$
s\left(x_{1}, \ldots, x_{n-1}\right)=t_{m}\left(x_{1}, \ldots, x_{n-1}, x_{n-1}\right)
$$

is absorbing for $\mathbf{B}$. The only non-trivial condition to verify is, $s\left(B, \ldots, B, B^{\alpha}\right) \subseteq B$. Let $b_{1}, \ldots, b_{n-2} \in B$ and $a \in B^{\alpha}$, and pick an arbitrary $b \in B$. Since $B^{2} \subseteq \alpha$, all elements $b_{1}, \ldots, b_{n-2}, a, b$ are mutually $\alpha$-equivalent, and so is the value $t_{m}\left(b_{1}, \ldots, b_{n-2}, b, a\right)$. Consider the equality

$$
t_{m}\left(b_{1}, \ldots, b_{n-2}, b, t_{m}\left(b_{1}, \ldots, b_{n-2}, b, a\right)\right)=t_{m}\left(b_{1}, \ldots, b_{n-2}, b, a\right) .
$$


Using abelianess at the $(n-1)$-th position, we replace $b$ for $a$ and obtain

$$
t_{m}\left(b_{1}, \ldots, b_{n-2}, a, t_{m}\left(b_{1}, \ldots, b_{n-2}, b, a\right)\right)=t_{m}\left(b_{1}, \ldots, b_{n-2}, a, a\right) .
$$

The left hand side of the equality is always in $B$, because $t_{m}$ is absorbing for $\mathbf{B}$, and so is the right hand side, equal to $s\left(b_{1}, \ldots, b_{n-2}, a\right)$. Notice that if $t$ is binary, we only used bin-abelianess.

Finally, consider an arbitrary $\alpha$-absorbing subalgebra $\mathbf{B}$ of $\mathbf{A}$ and put $B_{a}=B \cap\{a\}^{\alpha}$. Now $B_{a}$ is either empty, or it forms a subalgebra of $\mathbf{A}$, and in the latter case it is $\alpha$-absorbing, too: if $b_{1}, \ldots, b_{n-1} \in B_{a}$ and $c \in B_{a}^{\alpha}$, we have $t\left(b_{1}, \ldots, c, \ldots, b_{n-1}\right)$ both in $B$, because $t$ is absorbing for $\mathbf{B}$, and also in $\{a\}^{\alpha}$, because so are all arguments. Hence, $B_{a}$ is either empty, or $B_{a}=B_{a}^{\alpha}=\{a\}^{\alpha}$. So $B$ is a union of blocks of $\alpha$, hence $B^{\alpha}=B$. The same argument works for bin-absorption, too.

Lemma 4.2. Let $\mathbf{A}$ be a finite idempotent algebra and $\alpha$ a solvable (bin-solvable, resp.) congruence of $\mathbf{A}$. Then $\mathbf{A}$ does not have a proper $\alpha$-absorbing ( $\alpha$-bin-absorbing, resp.) subalgebra.

Proof. We proceed by induction on $k$, the degree of solvability of $\alpha$ over $0_{A}$ (i.e., $k$ is the length of the shortest solvable chain between $0_{A}$ and $\alpha$ ). If $k=1$, then $\alpha$ is abelian and Lemma 4.1 applies. Now, assume the statement holds for all algebras and their solvable congruences of degree $k$, and consider an algebra $\mathbf{A}$ and its solvable congruence of degree $k+1$. There is an abelian congruence $\beta \leq \alpha$ of $\mathbf{A}$ such that $\alpha$ is solvable over $\beta$ of degree $k$. According to Lemma 4.1, A has no proper $\beta$-absorbing subalgebra. By the induction assumption, $\mathbf{A} / \beta$ has no proper $\alpha / \beta$-absorbing subalgebra. Let $\mathbf{B}$ be an $\alpha$-absorbing subalgebra of $\mathbf{A}$. Then $\mathbf{B}$ is also $\beta$-absorbing in $\mathbf{A}$, hence $B=B^{\beta}$. Also, $\mathbf{B} / \beta$ is $\alpha / \beta$-absorbing in $\mathbf{A} / \beta$, hence $B / \beta=(B / \beta)^{\alpha / \beta}$. Both properties are satisfied only if $B=B^{\alpha}$. Similarly for the binary case.

Theorem 4.3. Let $\mathbf{A}$ be a finite idempotent algebra and $\alpha$ a solvable (bin-solvable, resp.) congruence of $\mathbf{A}$. Then $\mathbf{A}$ is $\alpha-H A F(\alpha-H B A F$, resp.).

Proof. Let $\mathbf{B}$ be a subalgebra of $\mathbf{A}$. Then $\left.\alpha\right|_{B}$ is a (bin-)solvable congruence of $\mathbf{B}$, and Lemma 4.2 applies.

Taking $\alpha=1_{A}$ and considering the idempotent reduct, we obtain Theorem 1.5 mentioned in the introduction.

Proof of Theorem 1.5. Let $\mathbf{A}$ be a finite (bin-)solvable algebra. Then its idempotent reduct $\mathbf{A}^{I}$ is also (bin-)solvable, hence HAF (HBAF). Now if $\mathbf{C}$ is a (bin-)absorbing subalgebra of $\mathbf{B} \leq \mathbf{A}$, then $\mathbf{C}^{I}$ is a (bin-)absorbing subalgebra of $\mathbf{B}^{I} \leq \mathbf{A}^{I}$, hence $C=B$.

4.2. Locally solvable $\Rightarrow \mathbf{H A F}$. Recall that a 2-snag is a polynomial subreduct isomorphic to the 2-element semilattice. A congruence $\alpha$ of an algebra $\mathbf{A}$ is called locally solvable if $\mathbf{A}$ has no 2-snag in an $\alpha$-block. An algebra $\mathbf{A}$ is locally solvable if $1_{A}$ is solvable.

From the tame congruence theory it follows (see [9, Theorem 7.2]) that a congruence of a finite algebra is solvable if and only if it is locally solvable. We are not able to prove this fact directly. However, we can prove an analogue of Theorem 4.3 for local solvability. The core of the argument is the following lemma. 
Lemma 4.4. Let $\mathbf{A}$ be a finite algebra with a proper absorbing subalgebra $\mathbf{B}$. Then $\mathbf{A}$ has a 2-snag $\mathbf{S}$ where $|S \cap B|=1$.

Proof. The proof is by induction on $|\mathbf{A}|$, thus we assume that the lemma holds for all the algebras with fewer elements.

Let $t$ an $n$-ary absorbing term operation for $\mathbf{B}$. We aim to reduce the arity of $t$ to $n=2$.

Using iteration in the last coordinate, as in the proof of Lemma 4.1, we can assume that $t$ satisfies

$$
t(\bar{x}, t(\bar{x}, y))=t(\bar{x}, y) .
$$

Suppose that $t(a, \ldots, a, b) \in B$ for some $b \in B, a \in A \backslash B$. The unary polynomial $f(x)=t(a, \ldots, a, x)$ satisfies $f^{2}=f$ and its image intersects both $B($ as $f(b) \in B)$ and $A \backslash B$ (as $f(a)=a)$. Then either $f=\mathrm{id}_{A}$, in which case $(B \cup\{a\} ; t(x, x, \ldots, x, y))$ is a polynomial subreduct of $\mathbf{A}$ whose basic operation is binary, idempotent, and absorbing for $\mathbf{B}$; or $f(A)$ is a proper subset of $A$, in which case $\mathbf{C}=(f(A) ; f t)$ is a smaller polynomial subreduct of A whose basic operation is idempotent and absorbing for a proper, nonempty subuniverse $f(A) \cap B$ of $\mathbf{C}$, and then we can use the induction assumption.

Now we can assume that $t(a, \ldots, a, b) \in A \backslash B$ for all $b \in B, a \in A \backslash B$. Choose $b \in B$ arbitrarily and consider the unary polynomial $f(x)=t(x, \ldots, x, b)$. This polynomial maps $B$ into $B$ (as $B$ is a subuniverse) and $A \backslash B$ to $A \backslash B$. There are two possibilities. If $f$ is a bijection then the inverse mapping $g$ is a polynomial and $\left(A ; g\left(t\left(x_{1}, \ldots, x_{n-1}, b\right)\right)\right)$ is a polynomial reduct whose basic operation is idempotent and absorbing for $\mathbf{B}$ and has arity $n-1$. If $f$ is not a bijection then a power $g$ of $f$ satisfies $g^{2}=g$ and $(g(A) ; g t)$ is a smaller polynomial subreduct whose basic operation is idempotent and absorbing for $f(A) \cap B$.

It remains to consider the case that $t$ is binary and satisfies $t(x, t(x, y))=t(x, y)$. The argument above proves that for every $a \in A \backslash B$ the unary polynomial $f(x)=t(a, x)$ is equal to the identity, therefore $t(a, x)=x$ for every $x \in A$. Now we iterate $t$ in the first variable, i.e.

$$
s(x, y)=\underbrace{t((t(t(x, y), y), \ldots), y)}_{m},
$$

so that $s(s(x, y), y)=s(x, y)$. This new operation still satisfies $s(a, x)=x$ for every $x \in A$ and $a \in A \backslash B$. For an arbitrary $b \in B, a \in A \backslash B$ we have $s(a, s(b, a))=s(b, a)$ and $s(s(b, a), a)=s(b, a)$. That means that $(\{s(b, a), a\} ; s)$ is a polynomial subreduct isomorphic to the 2-element semilattice - a 2-snag.

Recall that an algebra is locally finite if every finitely generated subalgebra is finite.

Theorem 4.5. Let $\mathbf{A}$ be a locally finite idempotent algebra and $\alpha$ a locally solvable congruence of $\mathbf{A}$. Then $\mathbf{A}$ is $\alpha-H A F$.

Proof. Local solvability carries to subalgebras, hence we only need to prove $\alpha$-absorptionfreeness. Suppose B is a proper $\alpha$-absorbing subalgebra, i.e., it is an absorbing subalgebra of $\mathbf{B}^{\alpha} \leq \mathbf{A}$. Choose an $\alpha$-block $\mathbf{C}$ such that $C \cap B$ is a proper subset of $C$. Let $\mathbf{D}$ be the subalgebra of $\mathbf{C}$ generated by an element in $B$ and an element in $C \backslash B$. Since $\mathbf{A}$ is locally finite, $\mathbf{D}$ is finite. By Lemma 4.4, $\mathbf{D}$ has a 2-snag, therefore $\alpha$ is not locally solvable.

We can formulate the following characterization of local solvability in terms of absorptionfreeness. 
Corollary 4.6. Let A be a locally finite algebra. The following are equivalent.

(1) A is locally solvable.

(2) All idempotent polynomial subreducts of $\mathbf{A}$ are absorption-free.

Proof. (2) $\Rightarrow(1)$ is trivial, since any 2 -snag is an idempotent polynomial subreduct with an absorbing element. (1) $\Rightarrow(2)$ follows from Theorem 4.5: If $\mathbf{B}$ is an idempotent polynomial subreduct of $\mathbf{A}$, it is also locally finite and locally solvable, and thus absorption-free.

We do not know whether there is a corresponding notion of local bin-solvability. Polynomial subreducts do not seem to work because a polynomial reduct of a bin-abelian algebra is not necessarily bin-abelian. Nevertheless, any term reduct is bin-abelian. Hence, bin-solvable algebras contain no 2-term-snag, i.e., a subreduct isomorphic to the 2-element semilattice. We do not now whether the converse could be true.

4.3. Solvability and Mal'tsev conditions. Combining the results we obtained, we get that finite (bin-)solvable algebras with a Taylor term also have a Mal'tsev term (cube term), see Corollary 1.6. One of the highlights of the tame congruence theory is a finer result $[9$, Theorem 9.6 (2) implies (6)].

Theorem 4.7. Let $\mathcal{V}$ be a locally finite variety with a Taylor term. Then

(1) there is a term which is Mal'tsev on blocks of locally solvable congruences in algebras in $\mathcal{V}$ (also found in [9]);

(2) there is a term which is Mal'tsev on blocks of solvable congruences of finite algebras in $\mathcal{V}$ (also found in [9]);

(3) there is a term which is cube on blocks of bin-solvable congruences of finite algebras in $\mathcal{V}$.

Proof. Again, we can assume that $\mathcal{V}$ is idempotent as we can replace $\mathcal{V}$ with its idempotent reduct. Corollary 3.3 provides a term $t$ that is a Mal'tsev term on every HAF algebra in $\mathcal{V}$. Let $\mathbf{A}$ be a finite algebra from $\mathcal{V}$, and $\alpha$ a solvable congruence of $\mathbf{A}$. According to Theorem 4.3, every block of $\alpha$ is HAF, and thus $t$ is a Mal'tsev term on it.

Similarly for bin-solvability and similarly for (possibly infinite) algebras A with a locally solvable congruence $\alpha$.

Parts (1), (2) were originally proved using the tame congruence theory. The proof has essentially two parts: first, possession of a Taylor term is equivalent to omitting type $\mathbf{1}$ (this is done in the proof of [9, Theorem 9.6]), and second, using the local structure of the other abelian type, type $\mathbf{2}$, a Mal'tsev term is obtained (the final assembly of the proof is done in [9, Theorem 7.12], with references throughout the first seven chapters of [9]). On the contrary, our new approach only relies on calculations in Section 4, in Theorem 3.2 and on the Absorption Theorem [1, Theorem 2.3].

4.4. An alternative proof of a partial converse to Theorem 4.7. In [9] it is proved that a converse to item (1) of Theorem 4.7 is true: If there is a Mal'tsev term on blocks of locally solvable congruences of algebras in $\mathcal{V}$ then $\mathcal{V}$ has a Taylor term. For idempotent locally finite varieties this implication follows easily from Taylor's theorem [20] which says that $\mathcal{V}$ has a Taylor term if and only if $\mathcal{V}$ does not contain an algebra whose every operation is a projection. 
We sketch a proof of "(1) implies Taylor" and "(3) implies Taylor" which uses confluent operations.

Let $\mathcal{V}$ be an idempotent, locally finite variety and assume that

- there is a term that is a Mal'tsev term on all blocks of every solvable congruence of every finite algebra in $\mathcal{V}$, or

- there is a term that is a cube term on all blocks of every bin-solvable congruence of every finite algebra in $\mathcal{V}$.

We will show that every finite algebra in $\mathcal{V}$ has a confluent term operation, hence Lemma 2.2 applies.

Let $\mathbf{A}$ be the smallest counterexample, that is, the smallest algebra in $\mathcal{V}$ with no confluent term operation. By the assumption A is non-abelian (otherwise we get a Malt'sev term, or a cube term which give us confluent operations). Straightforward arguments (as in Proposition 2.1 or Corollary 2.4) show that $\mathbf{A}$ is simple and that it is enough to find a term operation $t_{u, v}$ which satisfies the confluency condition for every $u, v$ with $\operatorname{Sg}_{\mathbf{A}}(\{u, v\})=\mathbf{A}$.

Let us denote $u=0$ and $v=1$ and assume $\operatorname{Sg}_{\mathbf{A}}(\{0,1\})=\mathbf{A}$. We aim to find $t_{0,1}$ as above. As $\mathbf{A}$ is non-abelian, there is a term operation $t\left(x_{1}, \ldots, x_{n}\right)$ of $\mathbf{A}$ such that:

$$
t\left(a, a_{2}, \ldots, a_{n}\right)=t\left(a, b_{2}, \ldots, b_{n}\right) \text { and } t\left(b, a_{2}, \ldots, a_{n}\right) \neq t\left(b, b_{2}, \ldots, b_{n}\right) .
$$

As $\mathbf{A}$ is idempotent and generated by $\{0,1\}$, by composing terms generating $a$ 's and $b$ 's and identifying coordinates, we obtain $t^{\prime}\left(x_{1}, \ldots, x_{8}\right)$ such that:

$$
\begin{aligned}
& t^{\prime}(0,0,1,1,0,0,1,1)=t^{\prime}(0,0,1,1,0,1,0,1), \\
& t^{\prime}(0,1,0,1,0,0,1,1) \neq t^{\prime}(0,1,0,1,0,1,0,1) .
\end{aligned}
$$

Finally, as $\mathbf{A}$ is simple we can further compose $t^{\prime}$ and then identify variables to obtain a term operation $t^{\prime \prime}$ such that $(\ddagger)$ holds for $t^{\prime \prime}$ and either $t^{\prime \prime}(0,1,0,1,0,0,1,1)$ or $t^{\prime \prime}(0,1,0,1,0,1,0,1)$ is equal to 1 .

Now, for a given term operation $s\left(x_{1}, \ldots, x_{m}\right)$ call a coordinate $i$ unimportant if we can find $a$ 's and $b$ 's such that:

$$
s\left(a_{1}, \ldots, a_{m}\right)=s\left(b_{1}, \ldots, b_{m}\right) \text { and } a_{i}=0 \text { and } b_{i}=1 .
$$

For $t^{\prime \prime}$, the coordinates 6 and 7 are unimportant by the first part of $(\ddagger)$, and the coordinates $1,3,5$ are unimportant since $t^{\prime \prime}$ is idempotent and one of two evaluations in ( $\ddagger$ ) is equal to 1 .

For a term operation $s\left(x_{1}, \ldots, x_{m}\right)$ define

$$
Q_{s}=\bigcap_{i-\text { important }} s(A, \ldots, A, 1, A, \ldots, A) .
$$

Note that $Q_{t^{\prime \prime}} \supseteq\{1, a\}$ for some $a \neq 1$.

Now let $t^{\prime \prime \prime}\left(x_{1}, \ldots, x_{n}\right)$ be a term operation with maximal (under inclusion) $Q_{t^{\prime \prime \prime}}$ which contains $\{1, a\}$. If $Q_{t^{\prime \prime \prime}} \ni 0$ then, by idempotency, $t^{\prime \prime \prime}$ can be taken for $t_{0,1}$ and the proof is finished. Suppose for a contradiction that $Q_{t^{\prime \prime \prime}} \varsubsetneqq A$. As $Q_{t^{\prime \prime \prime}}$ is not a union of congruence blocks in a congruence generated by $\{(1, a)\}$ there is a term operation $f(x, y, z)$ such that:

$$
\left[f(1,0,1) \in Q_{t^{\prime \prime \prime}} \text { and } f(a, 0,1) \notin Q_{t^{\prime \prime \prime}}\right] \text { or }\left[f(1,0,1) \notin Q_{t^{\prime \prime \prime}} \text { and } f(a, 0,1) \in Q_{t^{\prime \prime \prime}}\right] \text {. }
$$

It is straightforward to verify that the term operation $u=f * t^{\prime \prime \prime}$, the set $Q_{u}$ contains $Q_{t^{\prime \prime \prime}}$ as well as $f(a, 0,1)$ and $f(1,0,1)$, a contradiction. 


\section{Affine Representations}

5.1. Abelian and Taylor $\Rightarrow$ affine. An algebra is called affine, if it is polynomially equivalent to a module. H. P. Gumm and J. D. H. Smith discovered independently that the following conditions are equivalent for every algebra $\mathbf{A}$ :

(1) $\mathbf{A}$ is affine;

(2) $\mathbf{A}$ is abelian and has a Mal'tsev polynomial operation;

(3) A has a central Mal'tsev polynomial operation (centrality means that it is a homomorphism $\mathbf{A}^{3} \rightarrow \mathbf{A}$ ).

This fact is sometimes called the Fundamental Theorem on Abelian Algebras. The crucial implication is $(2) \Rightarrow(1)$, and its proof is fairly straightforward: pick 0 arbitrarily, define an abelian group on $A$ using the Mal'tsev polynomial in the obvious way, and consider the ring of unary polynomials preserving 0 . (Abelianess for terms of higher arities is crucial here, the property fails for bin-abelian algebras.) See, e.g., [16] for details. Now, Corollary 1.6 can be interpreted in the following way.

Theorem 5.1 (Hobby, McKenzie). Finite abelian algebras with a Taylor term are affine.

Recall that the original proof of the theorem used the tame congruence theory heavily (see the discussion below Theorem 4.7), and in the present paper, we provided a proof based on absorption theory. Yet another proof of Theorem 5.1 has been discovered recently by M. Stronkowski and published in [18]. It is based on the fact that finite algebras with a Taylor term have a weak near unanimity term [15]. If $w$ is such a term, consider a binary polynomial $x * y=w(x, y, c, \ldots, c)$, for an arbitrary $c$. Using abelianess, it is fairly easy to prove that $*$ is cancellative, see [18, Corollary 3.8], hence, in a finite algebra, it is a quasigroup operation. It follows that a Mal'tsev polynomial exists. Notice that this is a weaker result, as it produces a Mal'tsev polynomial only (not a term), and does not allow for a refinement, as in Theorem 4.7. Furthermore, the proof relies on existence of a weak near unanimity term. Its original proof by Maróti and McKenzie [15] is rather involved and uses the tame congruence theory. A proof based on absorption theory can be found in [1], yet the argument is far more complicated than the one behind the Absorption Theorem used in our new proof.

5.2. Quasi-affine representation. For abelian algebras that are non-Taylor, or infinite, we can consider a weaker affine representation. An algebra is called quasi-affine, if it is a polynomial subreduct of a module. Clearly, quasi-affine algebras are abelian. K. Kearnes and Á. Szendrei [11] proved that abelian algebras with a Taylor term are always quasi-affine, and they are affine under a somewhat stronger assumption that they satisfy an idempotent Mal'tsev condition that fails in semilattices. On the other hand, virtually nothing is known on quasi-affine representations of abelian non-Taylor algebras.

In the quest of finding whether Theorem 1.5 could be extended to infinite algebras, we can restrict to idempotent algebras: if there was a solvable algebra that is not HAF, its idempotent reduct had the same property. Here is an interesting observation:

Proposition 5.2. Idempotent quasi-affine algebras are $H A F$. 
Proof. It is easy to check that every idempotent quasi-affine algebra is in fact a subreduct of a module over a ring $R$, i.e., every term operation can be expressed as

$$
t\left(x_{1}, \ldots, x_{n}\right)=\sum_{i} r_{i} x_{i}
$$

where $r_{i} \in R$ such that $\sum r_{i}=1$. Subalgebras of quasi-affine algebras are quasi-affine too, so we only need to prove non-existence of a proper absorbing subalgebra in any idempotent quasi-affine algebra $A$. Let $B$ be an absorbing subalgebra, with an absorbing term $t\left(x_{1}, \ldots, x_{n}\right)=\sum_{i} r_{i} x_{i}$. Let $a \in A$ arbitrary. For every coordinate $j$ and every $b_{1}, \ldots, b_{n} \in B$, we get $r_{j} a+\sum_{i \neq j} r_{i} b_{i} \in B$, hence $r_{j} a \in B$ for every $j$, and thus also $a=\sum r_{j} a \in B$ because of idempotence. We obtained $B=A$.

In order to find an example of an idempotent abelian algebra that is not HAF, we need to consider an idempotent abelian algebra that is not quasi-affine. Surprisingly, no such example is known, and the problem to find one has appeared, for instance, on the list of open problems from the 2001 Szeged conference [22, Problem 10.5].

\section{REFERENCES}

[1] L. Barto, M. Kozik, Absorbing subalgebras, cyclic terms and the constraint satisfaction problem, Logical Methods in Computer Science 8/1:07 (2012), 1-26.

[2] L. Barto, M. Kozik, Constraint satisfaction problems solvable by local consistency methods, to appear in Journal of the ACM.

[3] C. Bergman, Universal algebra: Fundamentals and selected topics, Chapman \& Hall/CRC Press, 2011.

[4] J. Berman, P. Idziak, P. Marković, R. McKenzie, M. Valeriote and R. Willard, Varieties with few subalgebras of powers, Transactions of the American Mathematical Society, Vol. 362, Number 3, March 2010, 1445-1473.

[5] A. Bulatov, P. Jeavons, Algebraic structures in combinatorial problems, Technical Report MATH-AL-42001, Technische Universität Dresden, 2001.

[6] A. Bulatov, P. Jeavons, A. Krokhin, Classifying the complexity of constraints using finite algebras. SIAM J. Comput. 34 (2005), no. 3, 720-742.

[7] R. Freese, R. McKenzie, Commutator theory for congruence modular varieties. London Mathematical Society Lecture Note Series 125. Cambridge University Press, Cambridge, 1987.

[8] O. C. García, W. Taylor, The lattice of interpretability types of varieties. Mem. Amer. Math. Soc. 50 (1984), no. 305.

[9] D. Hobby, R. McKenzie, The structure of finite algebras, Contemporary Mathematics 76, American Mathematical Society, Providence, RI, 1988.

[10] P. Idziak, P. Marković, R. McKenzie, M. Valeriote, and R. Willard, Tractability and learnability arising from algebras with few subpowers, SIAM Journal on Computing, Vol. 39 (7) 2010, 3023-3037.

[11] K. Kearnes, Á. Szendrei, The relationship between two commutators, Internat. J. Algebra Comput. 8 (1998), no. 4, 497-531.

[12] K. Kearnes, Á. Szendrei, Clones of algebras with parallelogram terms, Internat. J. Algebra Comput. 22 (2012), 1250005.

[13] M. Kozik, A. Krokhin, M. Valeriote and R. Willard, Characterizations of several Maltsev conditions, submitted.

[14] P. Marković, M. Maróti, R. McKenzie, Finitely related clones and algebras with cube terms. Order 29 (2012), no. 2, 345-359.

[15] M. Maróti, R. McKenzie, Existence theorems for weakly symmetric operations. Algebra Universalis 59 (2008), no. 3-4, 463-489.

[16] R. McKenzie and J. Snow, Congruence modular varieties: commutator theory and its uses, in Structural theory of automata, semigroups, and universal algebra, NATO Sci. Ser. II Math. Phys. Chem. 207, 273329, Springer, Dordrecht, 2005. 
[17] J. Opršal, personal communication.

[18] M. Stronkowski, D. Stanovský, Embedding general algebras into modules. Proc. Amer. Math. Soc. 138 (2010), no. 8, 2687-2699.

[19] Á. Szendrei, Modules in general algebra, Contributions to general algebra 10, Heyn, Klagenfurt, 1998, pp. $41-53$.

[20] W. Taylor, Varieties obeying homotopy laws. Canad. J. Math. 29 (1977), no. 3, 498-527.

[21] R. Willard, A finite basis theorem for residually finite, congruence meet-semidistributive varieties, J. Symbolic Logic 65 (2000), 187-200.

[22] Sixty four problems in universal algebra, available at http://www.math.u-szeged.hu/confer/algebra/2001/progr

(Barto, Stanovský) Department of Algebra, Faculty of Mathematics and Physics, Charles University, Sokolovská 83, 18675 Praha 8, Czech Republic

(Kozik) Department of Theoretical Computer Science, Faculty of Mathematics and Computer Science, Jagiellonian University, Golebia 24, Kraków, Poland

E-mail address, Barto: barto@karlin.mff.cuni.cz

E-mail address, Stanovský: stanovsk@karlin.mff.cuni.cz

E-mail address, Kozik: marcin.kozik@uj.edu.pl 